\title{
TITLE: TOTAL AND UNBOUND PHENYTOIN LEVELS IN CRITICALLY ILL CHILDREN
}

AUTHORS: C D McClain MD, G Wolf MD PhD, B L. Dodson PharmD, M L. McManus MD $\mathrm{MPH}$

AFFILIATION: Department of Anesthesiology, Children's Hospital Boston and Harvard Medical School, Boston, MA, USA

INTRODUCTION: Phenytoin is a highly protein bound drug that is commonly used to treat and prevent seizures. The unbound fraction of phenytoin represents the active drug and is therefore of therapeutic interest. Commonly, total serum phenytoin concentrations are measured and used as surrogates for free levels in adjustment of dosing regimes. Because discrepancies between free and total levels may arise in certain patient populations, corrective algorithms, such as the Sheiner-Tozer equation have been proposed to predict unbound phenytoin levels in adult patients with abnormal albumin levels. However, because the unbound level of phenytoin is highly dependent on a number of factors in addition to serum albumin level, we hypothesized that total phenytoin levels might often be misleading in the care of critically-ill children. This study was undertaken to define the relationship between total and unbound phenytoin levels and determine the validity of Sheiner-Tozer equation in this patient population.

METHODS: A computerized medical record dataset was screened for patients in whom serum albumin and both free and total phenytoin levels were measured simultaneously from a single blood sample. The relationship between free and total phenytoin levels was then explored via linear regression (SPSS, Inc., Chicago) and the accuracy of the Sheiner-Tozer equation tested.

RESULTS: Between 2000 and 2004, eighty-nine patients were identified in whom complete results were available with ages ranging from 3 months to 34 years. Linear agreement between free and total phenytoin levels was found to be poor $(\mathrm{r}=0.6)$ and the Sheiner-Tozer equation was found to correctly predict free from total serum levels only $33.75 \%$ of the time.

DISCUSSION: In this population of children, there is a poor correlation between total and unbound fractions of phenytoin. Further, corrective algorithms, such as the Sheiner-Tozer equation, do little to improve this. Potential mechanisms for this include the influences of polypharmacy, altered drug metabolism, acid/base status and other factors beyond serum albumin levels. Use of measured total or corrected total phenytoin levels as a basis for treatment carry a high probability of under- or overdosing in this population. We therefore recommend the routine measurement of unbound phenytoin in critically ill children.

REFERENCES: Mlynarek ME, et al. Predicting Unbound Phenytoin Concentrations in the Critically Ill Neurosurgical Patient. Annals of Pharmacology 1996;30:219-23. 